\title{
The development and validation of ques- tionnaire to assess the collaboration and teamwork between doctors and nurses in public hospital in China
}

\author{
H Xu ${ }^{1}$, F Li $^{2}$, J M Johnston ${ }^{1}$ \\ ${ }^{1}$ School of Public Health, The University of Hong Kong \\ ${ }^{2}$ Guangzhou $8^{\text {th }}$ People Hospital
}

\begin{abstract}
This study reports the development and psychometric properties of a Chinese instrument to assess doctor-nurse teamwork and collaboration. 398 doctors and nurses participated in the study. Feedback from experts with international medical or nursing background and doctors and nurses working in hospitals in China informed the questionnaire refinement. Through validity and reliability test, a 26item questionnaire was developed. Three were identified. The overall questionnaire as well as each factor had high construct validity. The internal consistency reliability and test-retest reliability are also satisfactory.
\end{abstract}

Keywords: doctor-nurse collaboration, public hospital, questionnaire, validation

\section{Introduction}

Doctors and nurses have a long history of working together to resolve health care problems and meet health care needs of patients under their care. Although, the traditional hierarchical doctor-nurse relationship is still the norm, interprofessional collaborative relationships are becoming the more commonly defined mode of practice in many healthcare institutions ${ }^{[1,2]}$. However, this is not so characteristic in China where doctors and nurses in public hospitals have clearly defined and separate professional goals and roles ${ }^{[3-7]}$. As well, the Chinese healthcare system, though based on an insurance model, has a very different incentive structure to that found in western societies and much larger out-ofpocket co-payments. Such differences in role delineation and healthcare structure introduces many challenges to effective teamwork and collaboration ${ }^{[8]}$.

Much of the collaborative literature derives from and used tools designed for western healthcare settings. Therefore, in light of the differences between China hospital and Western's, the available tools may not have the psychometric properties necessary for measuring teamwork and collaboration between doctors and nurses working in a Chinese hospital setting.

\section{Aims and objectives}

This study aims to develop and validate a subjective measure of doctor-nurse collaboration and teamwork for use in public hospitals in China. 


\section{Methods}

\subsection{Setting and participants}

All the eligible doctors $(\mathrm{N}=156)$ and nurses $(\mathrm{N}=325)$ from a Guangzhou, China public hospital group (Total $\mathrm{N}=481$ ) were asked to participate in the study. The hospital group comprised two independent hospitals. The human resources department provided the unblinded researcher staff a list of all doctors and nurses working in both hospitals including name, sex, age and working title. Each staff member was assigned a unique study number from 001to 481 .

\subsection{Questionnaire development}

A three-step approach was used to identify and select items for the questionnaire. First, from a thematic review of the collaboration and teamwork literature and relevant published questionnaires $42 \mathrm{mul}$ tinomial categorical response (1 strongly disagree, 4 agree) items and 11 items requiring dichotomized and other responses were assembled.

\subsection{Process}

All eligible staff received an envelope, which contained a questionnaire (with their unique study number) and a consent form. The questionnaire took approximately 15 minutes to complete. If a subject could not be identified in 5 consecutive morning meetings they were removed from the recruitment pool. The questionnaire administration process took 5 days to complete. 2 weeks later, same participants have to complete the questionnaire again for test-retest use.

\subsection{Factor analysis}

Principal component analysis with orthogonal rotation (varimax), eigenval- ues $>1.0$ and factor loading score $\geq 0.4$ was used to sort items into factors. Three factors were extracted.

\subsection{Ethics}

Written consent was obtained from all subject participants. Ethics approval was obtained from the Institutional Review Board of the University of Hong Kong/Hospital Authority Hong Kong West Cluster (HKU/HA HKW IRB).

\section{Results}

A total of 398 of 466 (response rate 89.3\%) doctors and nurses completed the questionnaire. DH doctors had more work experience, and DH nurses were older, more likely to be married and had more work experience.

\subsection{Internal consistency}

The final questionnaire contained 26 multinomial response items. The scree plot, and eigenvalues supported a 3 -factor solution explaining $66.3 \%$ of the variance: factor 1: work related autonomy (10 items; Cronbach's alpha $=0.71)$; factor 2: work related skills (10 items, Cronbach's alpha $=0.88$ ); the factor 3: work related relationships (6 items, Cronbach's alpha $=0.73$ ). There was less than $2 \%$ nonresponse to individual scales items.

\subsection{Test-retest reliability}

Test-retest demonstrated acceptable scale reliability by ICC overall, for each hospital and healthcare professional group. JGH scores are slightly higher than DH; and nurses slightly higher than doctor's. But all the results are "good" for testretest reliability ${ }^{[6]}$. The test-retest response rate was $100 \%$. 


\section{Discussion}

The aim of this study was to examine the validity and reliability of 'work related collaboration scale' in two public hospitals in southern China. We set out to develop an instrument to assess doctors and nurses attitude to inter personal work relationships, skills, aptitudes and autonomy and how these impact teamwork and collaboration. To our knowledge this is the first Chinese language scale to assess work-related collaboration and teamwork attributes in both doctors and nurses. The pair-designed scale, enabled an understanding of the cross professional attitudes to work.

\section{Conclusion}

Collaboration and teamwork between doctors and nurses is very hard to measure and constrained by many cultural characteristics. The questionnaire we developed has a satisfactory reliability and validity in Chinese healthcare setting.

\section{References}

[1] Beuscart-Zephir, M. C., Pelayo, S., Anceaux, F., Maxwell, D., \& Guerlinger, S. (2007). Cognitive analysis of physicians and nurses cooperation in the medication ordering and administration process. International Journal of Medical Information, 76 Suppl 1, S65-77.

[2] Chaboyer, Wendy P., \& Patterson, Elizabeth. (2001). Australian hospital generalist and critical care nurses perceptions of doctor nurse collaboration. Nursing and Health Sciences, 3, 73-79. [3] Clarin, Olivia A. (2007). Strategies to Overcome Barriers to Effective Nurse Practitioner and Physician Collabora- tion. The Journal for Nurse Practitioners, 3(8), 538-548.

[4] Klipfel, J. M., Gettman, M. T., Johnson, K. M., Olson, M. E., Derscheid, D. J., Maxson, P. M., . . . Vierstraete, H. T. (2011). Using high-fidelity simulation to develop nurse-physician teams. Journal of Continuous Education Nursing, 42(8), 347-357; quiz 358-349.

[5] Lancaster, Tim, Dobbie, Wendy, Vos, Katrina, \& Yudkin, Patricia. (1999). Randomized trial of nurse assisted strategies for smoking cessation in primary care. British Journal of General Practice, 49, 191-194.

[6] Leever, A. M., Hulst, M. V., Berendsen, A. J., Boendemaker, P. M., Roodenburg, J. L., \& Pols, J. (2010). Conflicts and conflict management in the collaboration between nurses and physicians - a qualitative study. Journal of Interprofessional Care, 24(6), 612-624.

[7] O'Brien, J. L., Martin, D. R., Heyworth, J., \& Meyer, N. R. (2008). Negotiating transformational leadership: a key to effective collaboration. Nursing Health Science, 10(2), 137-143.

[8] Steinert, T., Goebel, R., \& Rieger, W. (2006). A nurse-physician coleadership model in psychiatric hospitals: results of a survey among leading staff members in three sites. International Journal of Mental Health Nursing, 15(4), 251-257. 